Original Research

\title{
Self Affirmation Reduces the Anxiety, LDH and Troponin I in the Clients with Coronary Heart Disease (CHD)
}

\section{Padoli Padoli $^{1}$, Joko Suwito ${ }^{1}$, and Tanto Hariyanto ${ }^{2}$}

${ }^{1}$ Faculty of Nursing Surabaya Health Ministry Polytechnic, Indonesia

${ }^{2}$ Faculty of Nursing Malang Health Ministry Polytechnic, Indonesia

\begin{abstract}
Introduction: Positive self affirmation is one of the psychological interventions that can be applied to the treatment of coronary heart disease; its effect is currently unknown. The purpose of this study was to prove the effect of self affirmation on anxiety, troponin I and LDH in coronary heart disease patients.
\end{abstract}

Methods: The type and design of the study was quasi-experimental with a nonrandomized post-test control group design. Thirty patients with coronary heart disease who were treated in the Camelia room of Dr Soetomo Hospital who had been selected were divided into 2 groups. The first group was given self affirmation twice / day for 20 minutes and the second group had standard care as the control group. After the intervention, anxiety measurements were taken, in addition to the measurement of troponin I and LDH.

Results: The results showed that self affirmation reduced anxiety $(\rho=0.03)$, decreased troponin I $(\rho=0.003)$ and decreased the lactate dehydrogenase (LDH) levels $(\rho=0.006)$.

Conclusion: Self-affirmation improves the client's emotions, preventing damage to the heart muscle cells. This is reflected by a decrease in the troponin I and LDH levels which are indicators of heart muscle damage.

\section{ARTICLE HISTORY}

Received: Dec 26, 2019

Accepted: Dec 31, 2019

\section{KEYWORDS}

self affirmation; anxiety; troponin I; LDH; coronary heart disease

\section{CONTACT}

Padoli Padoli1

$\triangle$ padolipolteksby@yahoo.co.id $\equiv$ Faculty of Nursing Surabaya Health Ministry Polytechnic, Indonesia

Cite this as: Padoli, P, Suwito, J, \& Hariyanto, T (2019). Self Affirmation Reduces the Anxiety, LDH and Troponin I in the Clients with Coronary Heart Disease (CHD). Jurnal Ners, 14(3si), 310-315 doi:http://dx.doi.org/10.20473/in.v14i3(si).17170

\section{INTRODUCTION}

Coronary Heart Disease (CHD) is the single largest cause of death in the United States, Western Europe and many other countries including Indonesia. Observational studies indicate that psychosocial factors strongly influence the development of coronary heart disease and that they influence the prognosis post-MI. Psychosocial factors in depression are a major problem in patients after they have experienced an acute heart attack, so psychological interventions specifically target these psychosocial risk factors to reduce the rate of recurrence of heart attacks (adverse cardiac events).

In Indonesia, the prevalence of CHD is increasing every year. A regular household health survey (SKRT) conducted by the Ministry of Health showed that heart disease contributed to $19.8 \%$ of all causes of death in 1993 . This number increased to $24.4 \%$ in 1998. The 2001 SKRT results for CHD ranked first in reference to the main causes of death in
Indonesia(Lestari, 2007). It is estimated that up to $20 \%$ of individuals experience depressive episodes within a few weeks of acute events. More than 25\% experience minor depression or dysrhythmias, with an odds ratio of 2 - 3 . The data of the patients treated in the Camelia Room in the RSUD year 2008 in the Dr. Soetomo Surabaya hospital showed 207 people (27.95\%) out of 741 cases of coronary heart disease wre diagnosed with IMA and in 2009, there were 243 (30.29\%) IMA patients out of 802 CHD patients. In 2010 during the period from January to September, the number of IMA patients numbered 199 people (35\%) out of 553 patients with CHD.

Psychological factors have been widely found to increase the risk and to worsen the prognosis of coronary disease. Increased corticosteroids due to stress will induce hypercholesterolemia, hypertriglyceridemia and hypertension. Other actions of induction due to atherosclerosis on steroids include injury to the endothelial blood vessel cells, intima and the inhibition of normal healing. 
Morning plasma cortisol increases significantly, which is correlated with moderate to severe coronary atherosclerosis. Sympatoadrenal hyperactivity contributes to the development of cardiovascular disease through the effects of catecholamines on the heart, blood vessels and platelets.

Some evidence suggests that the psychosocial factors in depression are a major problem in patients after experiencing an acute heart attack. Depressed patients experience several social problems during the first year of healing. They report an impaired quality of life, are less adherent to treatment and are slower to return to work than non-depressed patients. Several studies have shown that depression after an acute myocardial infarction is accompanied by an increase in the risk of future heart attacks and cardiac death(Kristina Orth-Gome, 2009). Stress and depression due to CHD attacks requires psychological interventions to prevent this risk and to improve the prognosis of coronary disease. Positive selfaffirmation interventions that control stress and that enhance the parasympathetic nervous system action are expected to reduce morbidity and to facilitate emotional and physical recovery after being discharged from the hospital. However, so far, apart from positive affirmations rarely being used, it has not been able to explain the improvement in healing in coronary heart disease patients.

The interaction of various factors can significantly speed up the disease process (Price, Silvia, 2006). Emotional factors and chronic stress experiences increase atherosclerosis and the risk of heart attacks. Acute emotional stress in mice causes increased creatinine kinase (CK) activity and increased alanine aminotransferase (ALT), aspartate aminotransferase (AST) and lactate dehydrogenase (LDH) in the plasma or serum. This increase in enzymes is thought to result from damage to the various tissues (Nutr et al., 2009). Acute anger is accompanied by twice the risk of myocardial infarction and mental stress, causing cardiac ischemia in $30-60 \%$ of patients with $\operatorname{CHD(Kop,Willem~J.,~}$ 2007).

Heart disease has a reciprocal relationship with emotional distress, psychosocial conditions and psychiatric disorders. The management of CHD is focused on risk factor modification intensively through drugs, changes in lifestyle, physical exercise and diet. The results revealed that around $75 \%$ of all physical diseases stem from mental and emotional problems. Unfortunately, most treatments rarely reach the source of this problem, namely the mind or emotions (W, 2007).

Cardiac rehabilitation services are comprehensive programs that involve education, training, risk factor modification and counseling, which are designed to limit the physiological and psychological effects of heart disease, reducing the risk of death or the recurrence of heart attacks, and increasing the client's psychosocial and vocational status(Contractor, 2011).
Self affirmation is very easy, fast and effective, reaching the subconscious mind, re-educating and healing the sick mind. Self affirmation is one of the psychological interventions that works to improve the work of the parasympathetic nervous system. Increased parasympathetic activity protects from the effects of electrical destabilization in the myocardium by increasing adrenergic tone. Mind relaxation also counters the high response due to stress. The relaxation of the mind as a physiological responses will affect the hypothalamic pituitary adrenal (HPA) axis and the sympathetic-adrenalmedullary (SAM) axis by decreasing the cortisol secretion in the adrenal gland of the cortex and decreasing the release of catecholamines through the sympathetic fibers in the medullary adrenal sympathetic axis. Decreased levels of cortisol and catecholamines will reduce cardiac demand, induce an increase in plasma volume, increase the mean heart variability (HRV), prevent coronary contraception and reduce platelet / plaque activation and rupturing related to atherosclerosis. The purpose of this study was to explain the effectiveness of self-affirmation related to anxiety, LDH and troponin I in the clients with coronary heart disease.

\section{MATERIALS AND METHODS}

Coronary Heart Disease (CHD) is the single largest cause of death in the United States, Western Europe and many other countries including Indonesia. Observational studies indicate that psychosocial factors strongly influence the development of coronary heart disease and that they influence the prognosis post-MI. Psychosocial factors in depression are a major problem in patients after they have experienced an acute heart attack, so psychological interventions specifically target these psychosocial risk factors to reduce the rate of recurrence of heart attacks (adverse cardiac events).

In Indonesia, the prevalence of CHD is increasing every year. A regular household health survey (SKRT) conducted by the Ministry of Health showed that heart disease contributed to $19.8 \%$ of all causes of death in 1993 . This number increased to $24.4 \%$ in 1998. The 2001 SKRT results for CHD ranked first in reference to the main causes of death in Indonesia(Lestari, 2007). It is estimated that up to $20 \%$ of individuals experience depressive episodes within a few weeks of acute events. More than 25\% experience minor depression or dysrhythmias, with an odds ratio of 2 - 3 . The data of the patients treated in the Camelia Room in the RSUD year 2008 in the Dr. Soetomo Surabaya hospital showed 207 people (27.95\%) out of 741 cases of coronary heart disease wre diagnosed with IMA and in 2009, there were 243 (30.29\%) IMA patients out of 802 CHD patients. In 2010 during the period from January to September, the number of IMA patients numbered 199 people (35\%) out of 553 patients with CHD.

Psychological factors have been widely found to increase the risk and to worsen the prognosis of 
coronary disease. Increased corticosteroids due to stress will induce hypercholesterolemia, hypertriglyceridemia and hypertension. Other actions of induction due to atherosclerosis on steroids include injury to the endothelial blood vessel cells, intima and the inhibition of normal healing. Morning plasma cortisol increases significantly, which is correlated with moderate to severe coronary atherosclerosis. Sympatoadrenal hyperactivity contributes to the development of cardiovascular disease through the effects of catecholamines on the heart, blood vessels and platelets.

Some evidence suggests that the psychosocial factors in depression are a major problem in patients after experiencing an acute heart attack. Depressed patients experience several social problems during the first year of healing. They report an impaired quality of life, are less adherent to treatment and are slower to return to work than non-depressed patients. Several studies have shown that depression after an acute myocardial infarction is accompanied by an increase in the risk of future heart attacks and cardiac death(Kristina Orth-Gome, 2009). Stress and depression due to CHD attacks requires psychological interventions to prevent this risk and to improve the prognosis of coronary disease. Positive selfaffirmation interventions that control stress and that enhance the parasympathetic nervous system action are expected to reduce morbidity and to facilitate emotional and physical recovery after being discharged from the hospital. However, so far, apart from positive affirmations rarely being used, it has not been able to explain the improvement in healing in coronary heart disease patients.

The interaction of various factors can significantly speed up the disease process (Price, Silvia, 2006). Emotional factors and chronic stress experiences increase atherosclerosis and the risk of heart attacks. Acute emotional stress in mice causes increased creatinine kinase (CK) activity and increased alanine aminotransferase (ALT), aspartate aminotransferase (AST) and lactate dehydrogenase $(\mathrm{LDH})$ in the plasma or serum. This increase in enzymes is thought to result from damage to the various tissues (Nutr et al., 2009). Acute anger is accompanied by twice the risk of myocardial infarction and mental stress, causing cardiac ischemia in $30-60 \%$ of patients with $\operatorname{CHD(Kop,Willem~J.,~}$ 2007).

Heart disease has a reciprocal relationship with emotional distress, psychosocial conditions and psychiatric disorders. The management of CHD is focused on risk factor modification intensively through drugs, changes in lifestyle, physical exercise and diet. The results revealed that around $75 \%$ of all physical diseases stem from mental and emotional problems. Unfortunately, most treatments rarely reach the source of this problem, namely the mind or emotions (W, 2007).

Cardiac rehabilitation services are comprehensive programs that involve education, training, risk factor modification and counseling, which are designed to limit the physiological and psychological effects of heart disease, reducing the risk of death or the recurrence of heart attacks, and increasing the client's psychosocial and vocational status(Contractor, 2011).

Self affirmation is very easy, fast and effective, reaching the subconscious mind, re-educating and healing the sick mind. Self affirmation is one of the psychological interventions that works to improve the work of the parasympathetic nervous system. Increased parasympathetic activity protects from the effects of electrical destabilization in the myocardium by increasing adrenergic tone. Mind relaxation also counters the high response due to stress. The relaxation of the mind as a physiological responses will affect the hypothalamic pituitary adrenal (HPA) axis and the sympathetic-adrenalmedullary (SAM) axis by decreasing the cortisol secretion in the adrenal gland of the cortex and decreasing the release of catecholamines through the sympathetic fibers in the medullary adrenal sympathetic axis. Decreased levels of cortisol and catecholamines will reduce cardiac demand, induce an increase in plasma volume, increase the mean heart variability (HRV), prevent coronary contraception and reduce platelet / plaque activation and rupturing related to atherosclerosis. The purpose of this study was to explain the effectiveness of self-affirmation related to anxiety, LDH and troponin I in the clients with coronary heart disease.

\section{RESULTS}

The characteristics of the research subjects in the control group were that most were women $(69,23, \%)$, almost half were unemployed or private employees $(23,08 \%)$, most suffered from an old miocardial infarction (OMI) $(46,15 \%)$ and the average age was 55.67 years. Most of the subjects in the treatment group were male $(64.71 \%)$, almost half were not working or they were private employees (29.41\%) and they had suffered from an acute myocardial infarction (IMA / 41.18\%) and OMI (41.18\%) (Table 1).

\section{Anxiety}

The mean anxiety score in the control group was 9.07 \pm 4.66 and in the treatment group it was $6.11 \pm 2.49$. The average anxiety scores in the treatment group were lower than in the control group. The data shows that self affirmation decreases anxiety more quickly than in the standard group of coronary heart disease clients (Table 2). The results of the independent $t$ test show a $\rho$ value $=0.033<\alpha=0.05$, therefore $\mathrm{H} 0$ is rejected. This means that there are significant differences in the level of anxiety between the clients who are given self-affirmation and standard care. 
Table . Distribution of the characteristics of the coronary heart disease clients

\begin{tabular}{|c|c|c|c|c|}
\hline Characteristics & Control Group & & ment G & \\
\hline Gender & f & $\%$ & f & $\%$ \\
\hline Man & 4 & 30,77 & 6 & 35,29 \\
\hline Women & 9 & 69,23 & 11 & 64,71 \\
\hline Total & 13 & 100,0 & 17 & 100,0 \\
\hline Work & f & $\%$ & f & $\%$ \\
\hline IRT/does not work & 3 & 23,08 & 5 & 29,41 \\
\hline Private & 6 & 46,15 & 7 & 41,18 \\
\hline Entrepreneurship & 2 & 15,38 & 1 & 5,88 \\
\hline Civil servants & 2 & 15,38 & 4 & 23,53 \\
\hline Total & 13 & 100,0 & 17 & 100,0 \\
\hline Medical diagnosis & $\mathbf{f}$ & $\%$ & $\mathbf{f}$ & $\%$ \\
\hline OMI & 6 & 46,15 & 7 & 41,18 \\
\hline NSTEMI & 2 & 15,38 & 1 & 5,88 \\
\hline ANGINA & 2 & 15,38 & 2 & 15,38 \\
\hline IMA & 3 & 23,08 & 7 & 41,18 \\
\hline Total & 13 & 100 & 17 & 100 \\
\hline Average age & 55,67 & years & 51,33 & Years \\
\hline
\end{tabular}

Table 2. Anxiety in the Coronary Heart Disease clients after being given self affirmation

\begin{tabular}{ccccc}
\hline Variables & Group & Means & SD & Sig. (2-tailed) \\
\cline { 1 - 3 } Anxiety & Control & 9,07 & $\pm 4,66$ & 0,03 \\
\cline { 2 - 4 } & treatment & 6,11 & $\pm 2,49$ & \\
\hline
\end{tabular}

Table 3. Troponin I in the Coronary Heart Disease clients after being given self affirmation

\begin{tabular}{ccccc}
\hline Variables & Groups & Means & SD & Sig. (2-tailed) \\
\cline { 1 - 4 } Troponin I & control & 15,39 & $\pm 16,92$ & 0,003 \\
\cline { 2 - 4 } & Treatment & 1,76 & $\pm 4,13$ & \\
\hline
\end{tabular}

Table 4. LDH value in the coronary heart disease clients after being given self affirmation

\begin{tabular}{ccccc}
\hline Variable & Groups & Means & SD & Sig. (2-tailed) \\
\cline { 2 - 4 } \multirow{2}{*}{ LDH } & Control & 905,33 & $\pm 652,26$ & 0,006 \\
& Treatment & 459,89 & $\pm 145,04$ & \\
\hline
\end{tabular}

When a threat or danger is perceived as excessive, the sympathetic nervous system input decreases and the parasympathetic system becomes more active. The 2 systems cannot operate together at the same time(Herbert, 1975)(Sapolsky, 1994a), so relaxation occurs when the sympathetic nervous system is more dominant. Nerve impulses originating from the cortex stimulate the hypothalamus where the parasympathetic response begins. The hypothalamus sends instructions through the nerves to the rest of the body. The vagus nerve is responsible for most of the innervation in the internal organs. The vagus nerve sends a message to slow down the heart rate, to lower the blood pressure, to stimulate digestion and to normalize the blood sugar levels. This response was identified by Herbert Benson as a relaxation response (Herbert, 1975).

\section{Troponin I}

The mean troponin I level in the control group was $15.39 \mathrm{ng} / \mathrm{ml} \pm 16.92$ and in the treatment group it was was $1.76 \mathrm{ng} / \mathrm{ml} \pm 4.13$. The mean troponin I level in the treatment group was lower than in the control group. The results of the independent $t$ test showed a value of $p 0.003>(p=0.05)$, meaning that there were differences in the levels of troponin I enzymes between the 2 groups, where for the coronary heart disease patients who got self-affirmation, the troponin I enzyme level tended to be lower than in the patients with standard care (Table 3).

\section{Lactate Dehydrogenase}

The mean LDH value of the control group was 905.33 \pm 652.26 and in the treatment group, it was $459.89 \pm$ 145.04. The results of the independent $t$ test showed a value of $p 0.006>(p=0.05)$, meaning that there were differences in the LDH enzyme levels between the two groups. The mean LDH in the treatment group was lower than in the control group. This shows that there are differences in the LDH values between the 2 groups. Where the coronary heart disease patients were given self-affirmation, the LDH enzyme values tended to be lower than in the patients with standard care (Table 4).

\section{DISCUSSION}

This study was a quasi-experimental study conducted on patients with coronary heart disease in the Camelia Room of Dr. Hospital. Soetomo Surabaya. The research design used was a post-test control group design because the researcher wanted to know the tendency of changes in anxiety, followed by the tendency of changes in heart cell function using the 
parameters of troponin I and LDH levels. Self affirmation iwas carried out twice a day for 20 minutes each session after the client returned from the ICCU room. the retrieval of data for the anxiety variable was carried out twice before and after the treatment, while the values for the Troponin I and LDH data were taken once, 3 days after giving the self affirmation in both the treatment and control groups.

\section{Anxiety}

Ischemic heart disease is a disease that causes death in developing countries throughout the world. Psychosocial factors are known today to play a significant and independent role in the development of ischemic heart disease and its complications. Mental stress has implications for triggering myocardial infarction (MI) and sudden death in patients with coronary artery disease[10]. A survey of the physical and psychological symptoms of CHD client anxiety shows that anxiety correlates with physical factors such as palpitations without exercise, anger, redness in the face, an abnormal pulse and muscle tension.

The psychological consequences of cardiovascular disease are known to affect recovery and quality of life. Anxiety and depression are the main consequences of the incidence of sudden death accompanied by CHD and cardiac arrhythmias (Roest, Martens, P, \& Denollet, 2010). Self-affirmation triggers a psychological cascade of effects, such as paying increasing attention to threats, broadening the perspective, increasing the feelings of social connection and enhancing their coping resources (Sapolsky, 1994b; Sherman \& Hartson, 2011).

The results of this study indicate that the average rating of anxiety in the client group given selfaffirmation (7.50) was lower than the group of clients who received standard care. Although the results of the statistical tests showed no difference in the groups $(\rho=0.169>a=0.05)$, the results indicated there to be a tendency for self affirmation to reduce anxiety in the clients owith coronary heart disease. Research states that self-affirmation plays an important role in increasing behavioral secretions (Stone et al., 1999) and it can reduce the defensive response to information that threatens their health.

The ventral striatum is a key region in the mesolimbic dopamine reward pathway which suggests that affirming important personal values is rewarding and it may lead to a cascade of effects associated with reward processing. Self-affirmation increases ventral striatum activity. Ventral striatum activity during self-affirmation activates a cascade of neural effects, including increased ventromedial prefrontal cortex activity, which in turn leads to psychological and behavioral changes. Selfaffirmation reduces the threat and stress responses, improves performance, reduces defensiveness and alters social and health behaviors

\section{Troponin I And Lactate Dehydrogenase (LDH)}

In myocardial necrosis, intracellular proteins will enter the interstitial space and go on to enter the systemic circulation via the local microvascular and lymphatic flow. Therefore, myocardial necrosis can be detected by examining the proteins in the blood caused by cell damage. These proteins include aspartate aminotransferase (AST), lactate dehydrogenase, creatine kinase isoenzyme MB (CK$\mathrm{MB}$ ), myoglobin, carbonic anhydrase III (CA III), myosin light chain (MLC) and cardiac troponin I and $\mathrm{T}$ (cTnI and cTnT). Increased serum levels of these proteins confirms the presence of myocardial infarction (Stürmer, Nigbur, Schacht, \& Sommer, 2011).

Some elevation of the cardiac troponin levels in patients with ST elevation MI, non-ST elevation MI, unstable angina, congestive heart failure and chronic renal insufficiency are indicative of poor outcomes (Sylvana, Fransisca, \& Da, 2005). Patients with a TnC elevation have higher mean cardiac mortality and they are more likely to have a coronary thrombus, more emboli in the coronary microvascular system and depression in their ventricular function (Van Koningsbruggen \& Das, 2008).

The results of this study indicate that selfAffirmation reduced the troponin I levels in the clients with coronary heart disease $(p=0.011)$, in addition to the level of the enzyme lactate dehydrogenase $(\mathrm{LDH})(\mathrm{P}=0.006)$. Decreased levels of this enzyme indicates an improvement in relation to the myocardial infarction risk in clients with coronary heart disease.

Self-affirmation relies on similar neural mechanisms to reduce the threat response. There is evidence that rewards and a positive effect increases the correlates of error-related negativity eventrelated potentials, which might relate to an improved conflict adaptation[18]. Self-affirming appears to encourage adaptive responses, reducing the responses that minimize the negative emotional impact of the information (fear control) and promoting responses that may eventually reduce the danger (danger control). In this case, it decreases anxiety, decreases the sympathetic activity and increases the parasympathetic response in CHD patients thereby reducing the risk of myocardial ischemia, which is reflected in a decrease in the troponin I and LHD levels.

\section{CONCLUSION}

Based on the results of the self-affirmation study to reduce anxiety, lactate dehydrogenase and troponin I in CHD clients, it can be concluded that self affirmation tends to reduce anxiety and prevent damage to the heart muscle cells. This is reflected by a decrease in the troponin I and lactac dehydrogenase (LDH) levels which are indicators of muscle damage in the heart. The results of this study suggest that self Affirmation should be carried out on CHD clients and that this can be initiated in the intensive care room after the patient's condition is stable until the healing period begins properly. Nurses need to be equipped with the knowledge of self affirmation techniques as 
a complementary therapy to provide effective patient services.

\section{REFERENCES}

Contractor, A. S. (2011). Cardiac Rehabilitation after Myocardial Infarction. 59(december), 51-55.

Herbert, B. (1975). The Relaxation Response. New York: Harper Collin Publisher Inc.

Kop,Willem J., et al. (2007). Psychoneuroimmunological Pathways Involved in Acute Coronary Syndromes. Psychoneuroimmunology.

Kristina Orth-Gome, N. S. H.-X. W. C. W. (2009). Stress Reduction Prolongs Life in Women With Coronary Disease. Circ Cardiovasc Qual Outcomes.

Lestari. (2007). Identifikasi Risiko dan Gejala Penyakit Jantung Koroner. Kantor Berita Indonesia, Gemari.

Nutr, J. C. B., Ohta, Y., Kaida, S., Chiba, S., Tada, M., Teruya, A., \& Imai, Y. (2009). Japan Stress in Increases in the Serum Levels Involvement of Oxidative of Various Enzymes and Components in Rats with Water-Immersion Restraint Stress. (November), 347-354.

Price, Silvia, et al. (2006). Patofisiologi: Konsep Klinis Proses-proses Penyakit (from Pathophysiology: Clinical Concepts of Disease Process). Jakarta: Penerbit Buku Kedokteran, EGC.

Roest, A. M., Martens, A. J., P, de J., \& Denollet, J.
(2010). Anxiety and risk of incident coronary heart disease: a meta-analysis. J Am Coll Cardiol, 1.

Sapolsky. (1994a). Why Zebras Don't Get Ulcers. New York: Henry Holt and Company.

Sapolsky. (1994b). Why Zebras Don't Get Ulcers. New York: Henry Holt and Company.

Sherman, D. ., \& Hartson, K. . (2011). Reconciling selfprotection with self-improvement: Selfaffirmation theory (In M. Alic). New York: Guilford Press.

Stone, P. H., Krantz, D. S., \& McMahon, R. P. (1999). Relationship among mental stress-induced ischemia and ischemia during daily life and during exercise.

Stürmer, B., Nigbur, R., Schacht, A., \& Sommer, W. (2011). Reward and punishment effects on error processing and conflict control. Frontiers in Psychology, 2. https://doi.org/10.3389/fpsyg.2011.00335

Sylvana, Fransisca, \& Da, G. (2005). Acute myocardial infarction. Wijaya Kusuma University Medical School.

Van Koningsbruggen, G. M., \& Das, E. (2008). Don't derogate this message! Self affirmation promotes online, type 2 diabetes risk test taking. Psychology and Health, 24, 635-649.

W, G. A. (2007). Hypnotherapy, The Art Subconsious Restructuring. Jakarta: Penerbit Gramedia Pustaka Utama. 